\title{
Comparison of diagnostic performance of four software packages for phase dyssynchrony analysis in gated myocardial perfusion SPECT
}

Koichi Okuda ${ }^{1 *}\left(\mathbb{D}\right.$, Kenichi Nakajima ${ }^{2 *}$, Shinro Matsuo ${ }^{2}$, Soichiro Kashiwaya ${ }^{3}$, Hiroto Yoneyama ${ }^{4}$, Takayuki Shibutani ${ }^{5}$, Masahisa Onoguchi ${ }^{5}$, Mitsumasa Hashimoto ${ }^{1}$ and Seigo Kinuya ${ }^{2}$

\begin{abstract}
Background: Phase analysis of gated myocardial perfusion single-photon emission computed tomography (SPECT) for assessment of left ventricular (LV) dyssynchrony was investigated using the following dedicated software packages: Corridor4DM (4DM), cardioREPO (cREPO), Emory Cardiac Toolbox (ECTb), and quantitative gated SPECT (QGS). The purpose of this study was to evaluate the normal values of 95\% histogram bandwidth, phase standard deviation (SD), and entropy and to compare the diagnostic performance of the four software packages. A total of 122 patients with normal myocardial perfusion and cardiac function (58.9 \pm 12.3 years, 60 women, ejection fraction (EF) $74.3 \pm 5.7 \%$, and end-diastolic volume (EDV) $83.5 \pm 3.6 \mathrm{~mL}$ ) and 34 patients with suspected LV dyssynchrony (64. $1 \pm 12.2$ years, 9 women, EF $52.0 \pm 18.0 \%$, and EDV $145.0 \pm 6.8 \mathrm{~mL}$ ) who underwent Tc-99m methoxy-isobutylisonitrile/tetrofosmin gated SPECT were retrospectively evaluated. Dyssynchrony indices of the $95 \%$ histogram bandwidth, phase SD, and entropy were computed with the four software programs. Diagnostic performance of LV phase dyssynchrony assessments was determined by receiver operator characteristic (ROC) analysis. The area under the ROC curve (AUC) was used to compare the software programs. The optimal cutoff point was determined by ROC curve based on the Youden index.
\end{abstract}

Results: The average of normal bandwidth significantly differed among the four software programs except in the comparison of 4DM and ECTb. Moreover, the normal phase SD significantly differed among the four software programs except in the comparison of CREPO and ECTb. The software programs showed high correlation levels for bandwidth, phase SD, and entropy $(r \geq 0.73, p<0.001)$. ROC AUCS of bandwidth, phase $S D$, and entropy were $\geq 0$. $850, \geq 0.858$, and $\geq 0.900$, respectively. Moreover, the ROC AUCs of bandwidth, phase SD, and entropy did not significantly differ among the four software programs. Optimal cutoff points for phase parameters were $24^{\circ}-42^{\circ}$ for bandwidth, $8.6^{\circ}-15.3^{\circ}$ for phase SD, and $31-48 \%$ for entropy.

Conclusions: Although the optimal cutoff value for determining LV phase dyssynchrony by ROC analysis varied depending on the use of the different software programs, all software programs can be used reliably for phase dyssynchrony analysis.

Keywords: Phase analysis, Left ventricular, Mechanical dyssynchrony, Myocardial perfusion SPECT, Cardiac resynchronization therapy

\footnotetext{
*Correspondence: okuda@kanazawa-med.ac.jp; nakajima@med.kanazawa-

u.ac.jp

'Department of Physics, Kanazawa Medical University, 1-1 Daigaku, Uchinada,

Kahoku, Ishikawa 920-0293, Japan

2Department of Nuclear Medicine, Kanazawa University Hospital, 13-1

Takara-machi, Kanazawa, Ishikawa 920-8641, Japan

Full list of author information is available at the end of the article
} 


\section{Background}

Cardiac resynchronization therapy (CRT) has been shown to be beneficial for patients with severe heart failure (HF) who do not respond to treatment with medications. The three eligibility criteria for CRT are a New York Heart Association (NYHA) functional class III or IV, left ventricular ejection fraction (LVEF) $\leq 35 \%$, and QRS duration $>120 \mathrm{~ms}$ in cardiac echocardiography $[1,2]$. Although patients with HF meet the eligibility criteria, one-third of patients did not respond to CRT $[3,4]$.

In order to improve the selection criteria for patients with HF to predict sufficient response to CRT, assessment of left ventricular (LV) mechanical dyssynchrony has been proposed. The LV mechanical dyssynchrony of patients with HF can be assessed using noninvasive imaging modalities such as an echocardiography with tissue Doppler imaging $[3,4]$ and a magnetic resonance imaging (MRI) $[5,6]$. Nuclear cardiology techniques, i.e., gated myocardial perfusion single-photon emission computed tomography (SPECT) (GMPS), have also been used to diagnose LV mechanical dyssynchrony [7-10]. Quantitative assessment of LV mechanical dyssynchrony by GMPS can be performed using commercially available software programs, including Emory Cardiac Toolbox (ECTb; Syntermed, Atlanta, GA, USA) [11], quantitative gated SPECT (QGS; Cedars-Sinai Medical Center, Los Angeles, CA, USA) [12], and Corridor4DM (4DM; INVIA Medical Imaging Solutions, Ann Arbor, MI, USA) [13] as well as cardioREPO (cREPO; FUJIFILM RI Pharma, Tokyo, Japan, developed in collaboration with EXINI Diagnosis, Lund, Sweden, and Kanazawa University, Kanazawa, Japan) [14-18]. The phase histogram and its distribution of the whole and regional LV can be automatically analyzed with these software programs, and these data can be utilized to diagnose LV dyssynchrony.

The purpose of this study was to clarify the normal values of 95\% histogram bandwidth, phase standard deviation (SD), and entropy and to compare the diagnostic performance of the following software programs in phase analysis for identifying LV phase dyssynchrony: 4DM, cREPO, ECTb, and QGS.

\section{Methods}

\section{Subjects}

A total of 156 patients, who underwent ${ }^{99} \mathrm{~m}$ Tc-sestamibi (MIBI) or ${ }^{99 \mathrm{~m}}$ Tc-tetrofosmin GMPS were retrospectively enrolled. Of these 156 patients, 122 were diagnosed as having normal perfusion (summed stress score $\leq 3)$ and cardiac function $(E F \geq 50 \%)$. The data for these patients were included in a Japanese normal database generated by the Japanese Society of Nuclear Medicine working group (JSNM-WG). The patient selection criteria for generating the normal database have been summarized elsewhere [19-22]. The remaining 34 patients who underwent GMPS in Kanazawa University Hospital were indicated for the determination of CRT implantation $(n=15)$, screening for the left bundle branch block (BBB) $(n=15)$, right $\mathrm{BBB}(n=3)$, and pulseless ventricular tachycardia $(n=$ 1). Of 34 patients, 15 patients were diagnosed as having heart failure with NYHA class III or IV symptoms. These clinical diagnoses were made separately in the Department of Cardiology. Consequently, we used these clinical diagnoses for discrimination between normal and abnormal phase distributions in GMPS data. The patient characteristics are shown in Table 1. Informed consent was obtained from all patients in the hospital. The ethics committee of Kanazawa University approved this study.

\section{Image acquisition}

With regard to the image acquisition condition for patients with suspected LV dyssynchrony, GMPS was performed with a dual-head gamma camera (Symbia T6 hybrid SPECT/CT scanner, Siemens Japan, Tokyo, Japan) equipped with a low-energy high-resolution (LEHR) collimator. A photopeak window of ${ }^{99 \mathrm{~m}} \mathrm{Tc}$ was set as a $15 \%$ energy window centered at $140 \mathrm{keV}$. The acquisition pixel size was $6.6 \mathrm{~mm}$ in a $64 \times 64$

Table 1 Characteristics of the study population

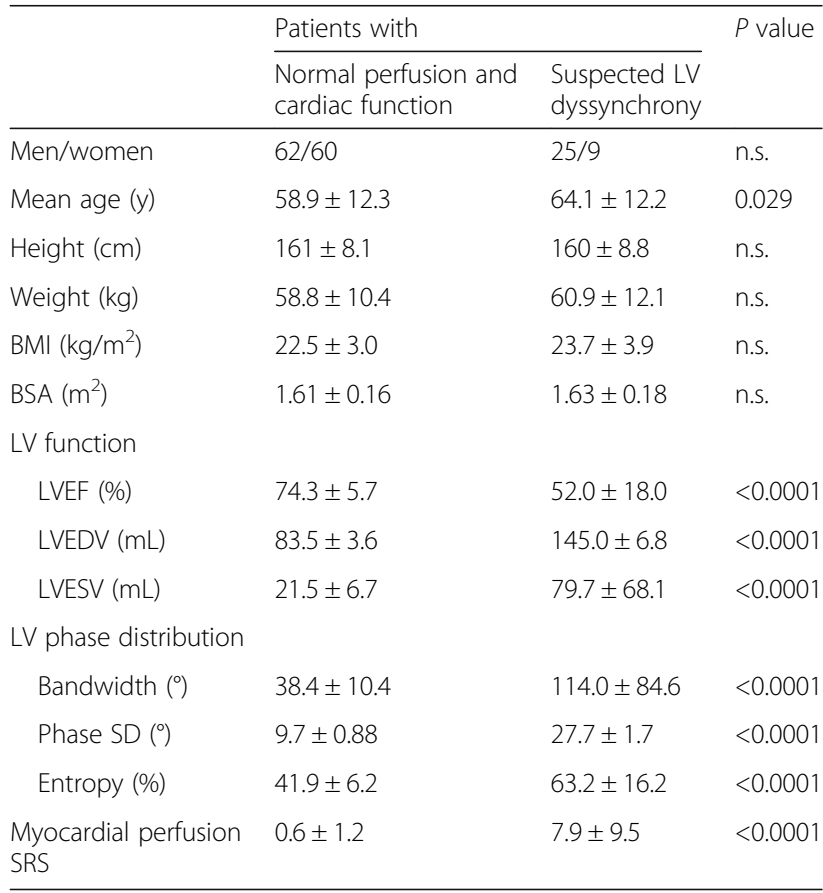

LVEF, LVEDV, LVESV, bandwidth, phase SD, and entropy were calculated with a cardioREPO software program. SRS was calculated with a quantitative perfusion score (QPS) software program

Abbreviations: $B M I$ body mass index, $B S A$ body surface area by the DuBois formula, $L V$ left ventricular, $E F$ ejection fraction, $E D V$ end-diastolic volume, $E S V$ end-systolic volume, SD standard deviation, SRS summed rest score 
matrix. Sixteen frames per cardiac cycle were used during data acquisition in GMPS. A circular orbit of the gamma cameras was adopted for $360^{\circ}$ SPECT image acquisition with 60 projections. ${ }^{99 \mathrm{~m}} \mathrm{Tc}-\mathrm{MIBI}$ or ${ }^{99 \mathrm{~m}} \mathrm{Tc}$-tetrofosmin of $300-370 \mathrm{MBq}$ was injected intravenously. The time per view of the SPECT acquisitions were $35 \mathrm{~s}\left(360^{\circ}\right.$ acquisition with 60 views), $50 \mathrm{~s}\left(180^{\circ}\right.$ acquisition with 32 views $)$, and $60 \mathrm{~s}\left(180^{\circ}\right.$ acquisition with 30 views). The detailed acquisition conditions for the JSNM-WG normal database have been summarized elsewhere [19-22].

\section{Image analysis}

The LVEF, end-diastolic volume (LVEDV), and endsystolic volume (LVESV) were automatically calculated with a CREPO software program. A histogram of phase distribution in the whole LV was computed from rest GMPS data. The phase SD and bandwidth were calculated with 4DM, cREPO, ECTb, and QGS programs. The bandwidth was expressed as $95 \%$ width of the phase histogram [7]. Entropy of the histogram distribution was calculated with QGS and cREPO because of unavailability in 4DM and ECTb [12, 19, 23]. Summed rest score (SRS) was calculated with a quantitative perfusion score (QPS; Cedars-Sinai Medical Center, Los Angeles, CA, USA) software program [12].

\section{Statistical analysis}

All continuous values are expressed as a mean \pm standard deviation. Student $t$ test was used to analyze the differences among continuous variables. For the paired continuous variables, a paired $t$ test was used to analyze the differences. Linear regression analysis was used to explain the relationship between two continuous variables. The Tukey-Kramer method was used for multiple comparisons of the bandwidth and phase SD among software programs. The Bland-Altman analysis was used for the assessment of the agreement. The receiver operating characteristic (ROC) area under the curve (AUC) values were expressed as mean \pm standard error of the mean. Subsequently, the optimal cutoff points were determined by ROC curve based on the Youden index [24]. All statistical tests were two-tailed, and the $p$ values $\leq 0.05$ were considered significant. These analyses were performed using MedCalc version 14.12.0 (MedCalc Software bvba, Ostend, Belgium) and JMP version 11.2.1 (SAS Institute Inc., Cary, NC, USA).

\section{Results}

\section{Study population}

The mean age of patients with suspected LV dyssynchrony was slightly higher than that of those with normal perfusion (64.1 vs. 58.9 years, $p=0.029)$. With regard to LV function and phase distribution, the LVEF significantly decreased, and LVEDV, LVESV, bandwidth, phase SD, and entropy significantly increased in patients with suspected LV dyssynchrony in comparison with normal patients $(p<0.0001)$. The patients with suspected LV phase dyssynchrony had moderate myocardial perfusion abnormalities (mean SRS; 7.9 \pm 9.5 ). When we calculated LVEF values in 15 patients with HF and 19 patients with echocardiographic abnormality, mean EF values were $38 \pm 14 \%$ for those with $\mathrm{HF}$ and 64 $\pm 11 \%$ for those with electrocardiographic abnormality $(p<0.0001)$. Thus, this result showed that the patients with suspected LV dyssynchrony had slight to severe cardiac function abnormalities.

\section{Phase distribution}

The phase distribution of patients with normal perfusion is shown in Fig. 1. The mean bandwidth significantly differed among the four software programs (QGS, 20.5 $\pm 7.8^{\circ} ; \mathrm{ECTb}, 28.1^{\circ} \pm 9.1^{\circ} ; 4 \mathrm{DM}, 29.6^{\circ} \pm$ $9.3^{\circ}$; and cREPO, $38.4^{\circ} \pm 10.4^{\circ} ; p<0.0001$ for all combinations except the combination of ECTb and 4DM $(p=\mathrm{n} . \mathrm{s})$.$) . The mean phase SD also significantly dif-$ fered among the four software programs (QGS, $5.7^{\circ}$ $\pm 4.4^{\circ} ; \mathrm{ECTb}, \quad 10.4^{\circ} \pm 4.8^{\circ} ; \quad 4 \mathrm{DM}, \quad 7.5^{\circ} \pm 2.3^{\circ} ; \quad$ and cREPO, $9.7^{\circ} \pm 2.8^{\circ} ; p<0.0001$ for all combinations except the combination of QGS and 4DM ( $p=$
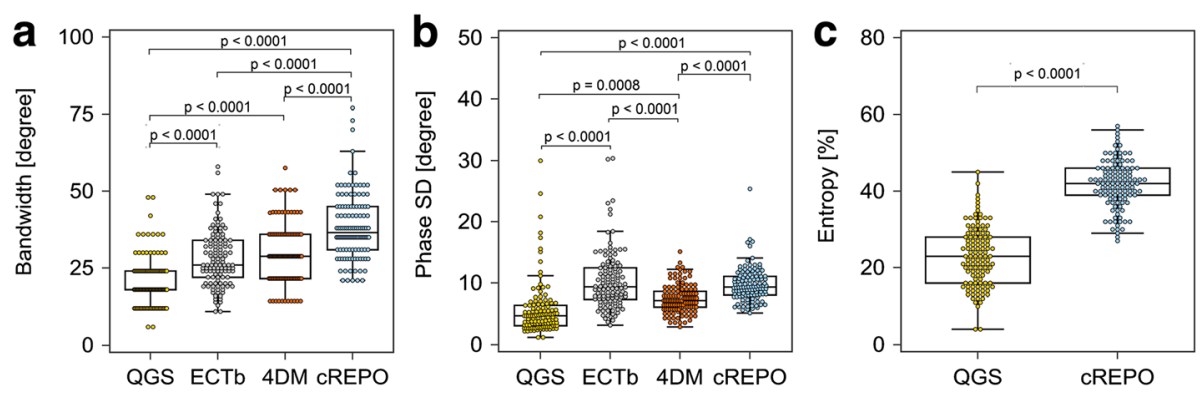

Fig. 1 The box-and-whisker plots of bandwidth (a), phase SD (b), and entropy (c) in patients with normal perfusion and cardiac function ( $n=122)$. These phase parameters were computed with QGS, ECTb, 4DM, and CREPO. SD standard deviation, QGS quantitative gated SPECT, ECTb Emory Cardiac Toolbox, 4DM Corridor 4DM, cREPO cardioREPO 
0.008), and ECTb and cREPO $(p=n$. s.)). A significant difference was observed between the mean entropies calculated by QGS and cREPO $(23.0 \pm 7.7 \%$ vs. $41.9 \pm 6.2 \%, p<0.0001)$.

The phase distribution of patients with suspected LV dyssynchrony is shown in Fig. 2. There were no significant differences in bandwidth among the four software programs (QGS, 75.9 $\pm 50.3^{\circ}$; ECTb, $90.6^{\circ} \pm 65.1^{\circ}$; $4 \mathrm{DM}$, $107.0^{\circ} \pm 86.3^{\circ}$; and $\left.\mathrm{cREPO}, 114.0^{\circ} \pm 84.6^{\circ}\right)$. There were also no significant differences in phase $\mathrm{SD}$ among the four software programs (QGS, $22.1^{\circ} \pm 15.6^{\circ}$; ECTb, $29.8^{\circ}$ $\pm 20.0^{\circ} ; 4 \mathrm{DM}, 26.7^{\circ} \pm 21.7^{\circ} ;$ and $\mathrm{cREPO}, 27.7^{\circ} \pm 20.3^{\circ}$. Significant differences in entropy were observed between QGS and cREPO $(50.0 \pm 16.8 \%$ vs. $63.2 \pm$ $16.2 \%, p<0.0001)$.

\section{Correlation and agreement between software programs}

The relationship and agreement among bandwidths obtained from the four software programs are shown in Fig. 3. The bandwidths obtained by ECTb was highly correlated with those obtained by 4DM $(r=0.87)$, cREPO $(r=0.93)$, and QGS $(r=0.83)$. The Bland-Altman analysis revealed that the mean differences were $4.7^{\circ}$, $13.1^{\circ}$, and $-9.2^{\circ}$ for $4 \mathrm{DM}$ vs. ECTb, cREPO vs. ECTb, and QGS vs. ECTb, respectively. The 95\% limits of agreement (LoA) between cREPO and ECTb was narrower than those between $4 \mathrm{DM}$ and $\mathrm{ECTB}$, and between QGS and ECTb.

The relationship and agreement among phase SDs obtained from the four software programs are shown in Fig. 4. The phase SDs obtained by ECTb was highly correlated with those obtained by 4DM $(r=$ $0.83)$, cREPO $(r=0.79)$, and QGS $(r=0.73)$. The Bland-Altman plots of the three software programs based on ECTb showed negative mean differences of $-2.9^{\circ},-1.0^{\circ}$, and $-5.3^{\circ}$ for $4 \mathrm{DM}$, cREPO, and QGS, respectively. The 95\% LoA of QGS exhibited slightly wide variation in comparison with 4DM and cREPO.

The relationship and agreement between entropies obtained from QGS and cREPO are shown in Fig. 5.
Although the correlation coefficient was good $(r=0.82)$, the linear regression line was shifted to the right by 16.6\%. The Bland-Altman analysis revealed that the mean of the difference was $-17.7 \%$ and the $95 \%$ LoA ranged from -34.7 to $-0.6 \%$.

\section{ROC analysis}

The ROC curve and its AUC are shown in Fig. 6. There were no significant AUC differences in bandwidths derived from the four software programs (QGS, $0.916 \pm 0.033$; ECTb, $0.883 \pm 0.038 ; 4 \mathrm{DM}, 0.888$ \pm 0.038 ; and CREPO, $0.850 \pm 0.044$ ). The sensitivity, specificity, and accuracy are shown in Fig. 7. The sensitivity, specificity, and accuracy of the bandwidth ranged from 79 to $82 \%, 71$ to $86 \%$, and 77 to $85 \%$, respectively. The lowest and highest cutoff points of bandwidth were $24^{\circ}$ for QGS and $42^{\circ}$ for cREPO, respectively, as observed in Table 2. When the optimal cutoff points of bandwidth for the four software programs were used, percentages of patients who were classified differently were $15 \%$ for $4 \mathrm{DM}, 28 \%$ for cREPO, 18\% for ECTb, and 15\% for QGS.

There were also no significant differences in AUC analysis in terms of the phase SD derived from the four software programs (QGS, $0.877 \pm 0.039$; ECTb, $0.858 \pm 0.042 ; 4 \mathrm{DM}, 0.893 \pm 0.035$; and cREPO, 0.884 \pm 0.036 ). The sensitivity, specificity, and accuracy of the phase SD ranged from 76 to $79 \%, 83$ to $89 \%$, and 80 to $84 \%$, respectively. The lowest and highest cutoff points of phase SD were $8.6^{\circ}$ for QGS and $15.3^{\circ}$ for $\mathrm{ECT} \mathrm{b}$, respectively. The percentages of patients who were classified differently using cutoff points for phase SD were $18 \%$ for $4 \mathrm{DM}, 18 \%$ for cREPO, $21 \%$ for ECTb, and 14\% for QGS.

No significant difference in AUC of entropy was observed between QGS $(0.915 \pm 0.034)$ and cREPO $(0.900 \pm 0.034)$. The sensitivity, specificity, and accuracy of an entropy were 82,87 , and $85 \%$ for QGS, and 79,87 , and $83 \%$ for cREPO, respectively. The cutoff point of entropy obtained by cREPO was higher than
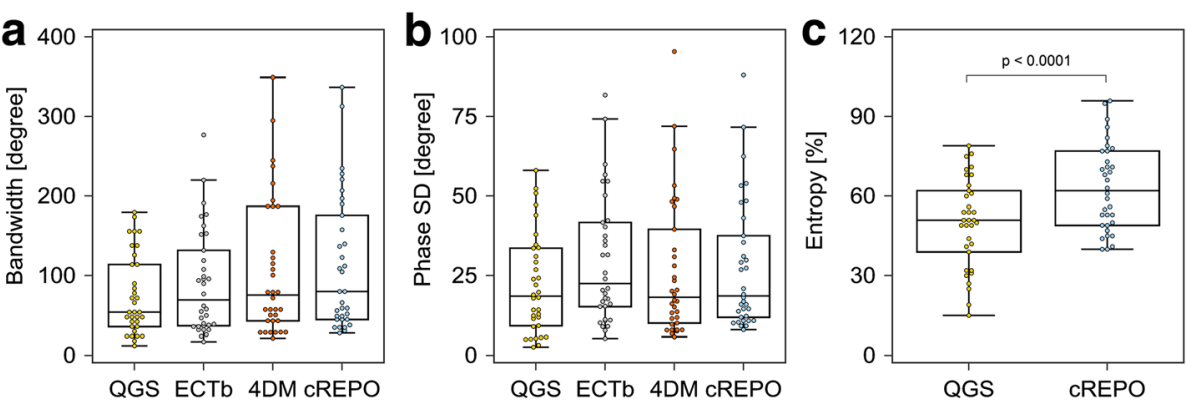

Fig. 2 The box-and-whisker plots of bandwidth (a), phase SD (b), and entropy (c) in patients with suspected LV dyssynchrony $(n=34)$. These phase parameters were computed with QGS, ECTb, 4DM, and CREPO. SD standard deviation, LV left ventricular, QGS quantitative gated SPECT, ECTb Emory Cardiac Toolbox, 4DM Corridor 4DM, CREPO cardioREPO 

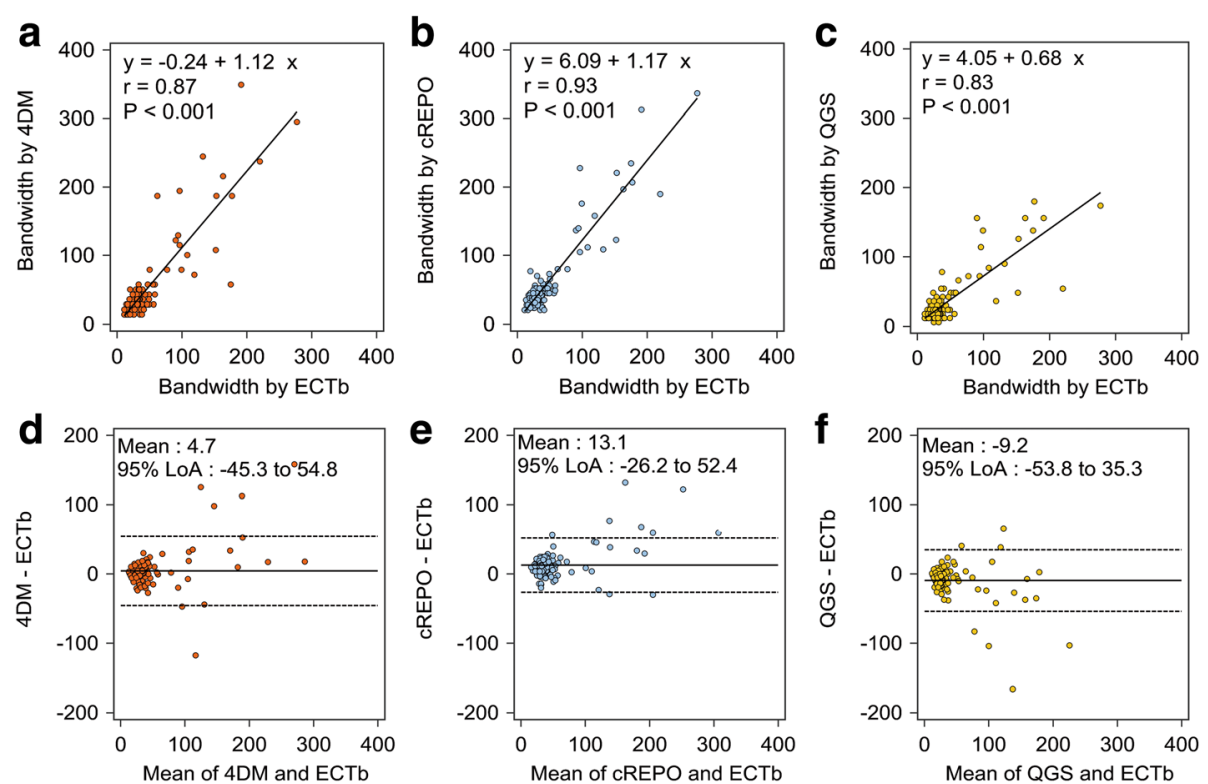

Fig. 3 Scatter diagrams with regression line of bandwidth between 4DM and ECTb (a), CREPO and ECTb (b), and QGS and ECTb (c). The BlandAltman plots of bandwidth between 4DM and ECTb (d), CREPO and ECTb (e), and QGS and ECTb (f). Continuous lines and dashed lines denote the mean difference between bandwidths by two software programs and upper and lower 95\% limits of agreement, respectively. QGS quantitative gated SPECT, ECTb Emory Cardiac Toolbox, 4DM Corridor 4DM, cREPO cardioREPO

that obtained by QGS. The percentages of patients who were classified differently using cutoff points for entropy were $15 \%$ for cREPO and $14 \%$ for QGS.

The diagnostic performance of the bandwidth assessment was equivalent to that of the phase SD in $4 \mathrm{DM}$,
cREPO, and ECTb except for QGS (AUC; 0.916 vs. 0.877, $p=0.0047)$. Regarding the phase entropies obtained by QGS and cREPO, the ROC AUC of entropy showed significantly higher values than that of bandwidth in cREPO $(p=0.008)$ and that of phase SD in QGS $(p=0.016)$.
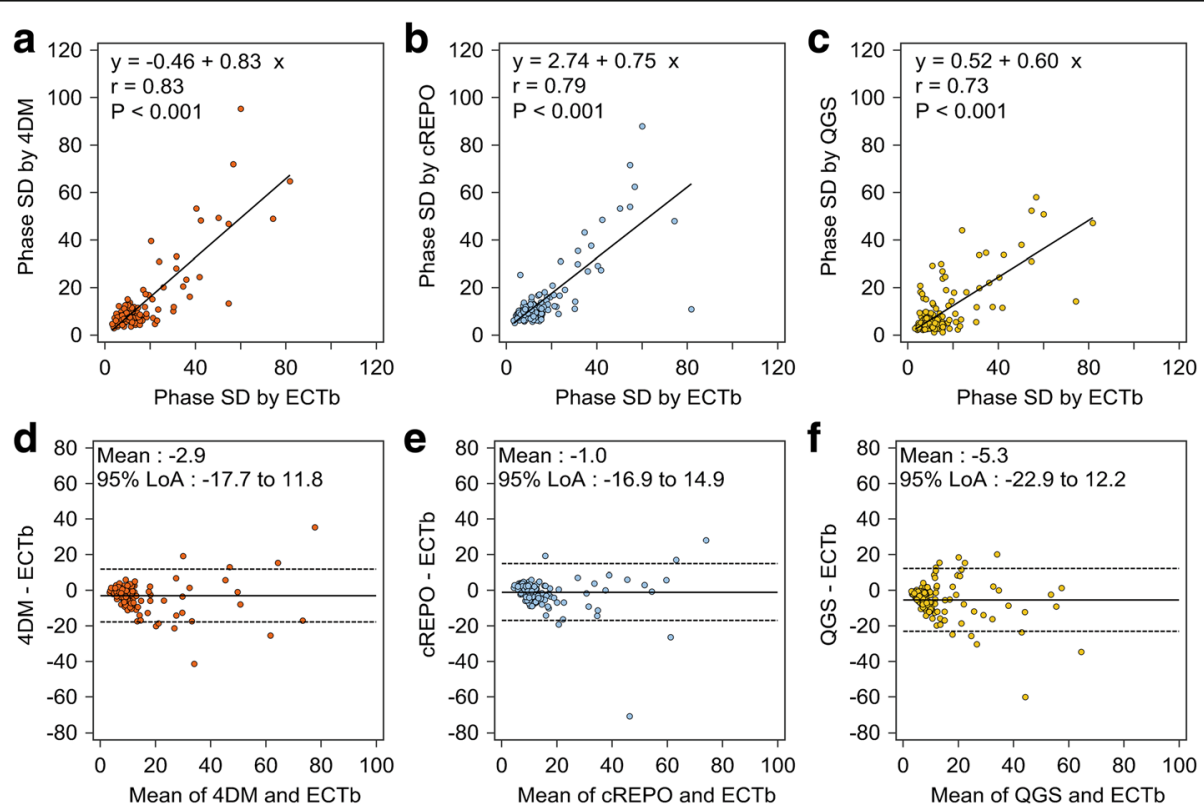

Fig. 4 Scatter diagrams with regression lines of phase SD between 4DM and ECTb (a), CREPO and ECTb (b), and QGS and ECTb (c). The Bland-Altman plots of phase SD between 4DM and ECTb (d), CREPO and ECTb (e), and QGS and ECTb (f). Continuous lines and dashed lines denote the mean difference between phase SDs by two software programs and upper and lower $95 \%$ limits of agreement, respectively. SD standard deviation, QGS quantitative gated SPECT, ECTb Emory Cardiac Toolbox, 4DM Corridor 4DM, CREPO cardioREPO 


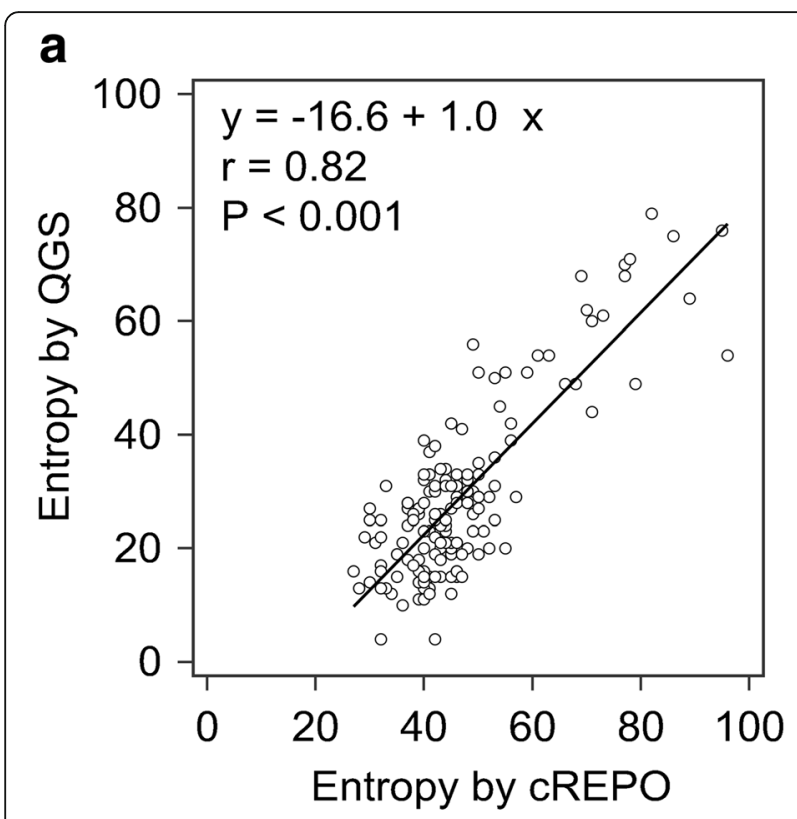

b

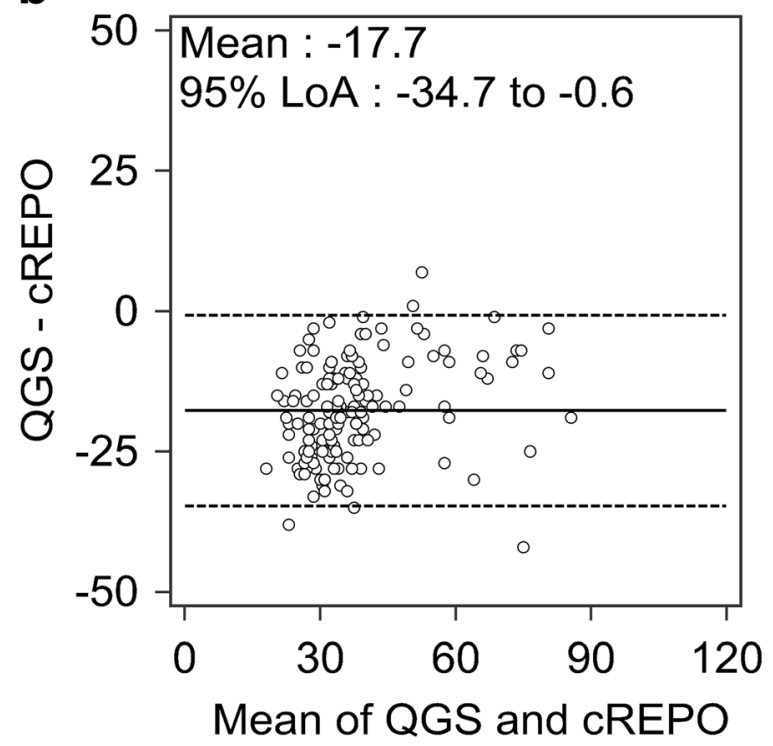

Fig. 5 Scatter diagram with regression line of entropy between QGS and CREPO (a). The Bland-Altman plot of entropy between QGS and CREPO (b). Continuous line and dashed lines denote the mean difference between entropies by QGS and CREPO, and upper and lower 95\% limits of agreement, respectively. QGS quantitative gated SPECT, ECTb Emory Cardiac Toolbox, 4DM Corridor 4DM, CREPO cardioREPO

\section{Discussion}

The main purpose of this study was to evaluate the diagnostic performance of four software packages: 4DM, cREPO, ECTb, and QGS in phase analysis. The relationship and agreement of phase parameters obtained from the four software programs were good. We could clearly determine that all software programs may be used reliably for phase analysis.

\section{Software algorithm for calculating LV dyssynchrony}

Our results demonstrated that the cutoff values of bandwidth, phase SD, and entropy for evaluating LV dyssynchrony were variable depending on the software programs. The cause of these differences might be based on the delineation of the LV contour and the bin size of a phase histogram. Regarding the delineation of the LV contour, edge detection of the basal LV myocardium would play an important role in creation of the histogram for phase distribution. ECTb performs the function of excluding outliers of bandwidth and phase SD by smoothing the phase array data on a polar map. When the outliers of bandwidth and phase SD are observed in the aortic valve area or basal area of the polar map, we can manually exclude the aortic and basal areas from the phase calculation in cREPO. According to the bandwidth in Fig. 1a, the four software programs output discrete data of $6^{\circ}$ for QGS, $1^{\circ}$ for ECTb, $7^{\circ}$ for $4 \mathrm{DM}$, and $3^{\circ}$ for cREPO. This result demonstrated that the bin sizes of a phase histogram differed in each software program.

\section{Normal phase distribution}

With regard to normal phase distributions in previously reported studies, the mean bandwidth ranged from $27.9^{\circ}$ to $42.0^{\circ}$ and the mean phase $\mathrm{SD}$ ranged from $8.6^{\circ}$ to $15.7^{\circ}$ in ECTb $[7,25-28]$. AIJaroudi et al. reported mean phase SDs of $6.1^{\circ}$ in a stress condition and $10.2^{\circ}$ at rest in 4DM [29]. QGS exhibited a mean bandwidth of $30.9^{\circ}-80.2^{\circ}$, mean phase $\mathrm{SD}$ of $10^{\circ}-22.2^{\circ}$, and mean entropy of $46.3-56.6 \%[23,30]$. Nakajima et al. have reported a mean bandwidth of $40^{\circ}$, mean phase SD of $10^{\circ}$, and mean entropy of $43 \%$ in cREPO. In comparison with our results, both mean bandwidth and phase SD calculated by QGS showed larger values in the reported studies.

\section{Which parameters are the best for phase analysis?} In analyses of phase distribution derived from GMPS, the $95 \%$ bandwidth, phase SD, entropy, kurtosis, and skewness are available using commercially software packages. Romeo-Farina reported that both bandwidth and phase SD showed excellent ROC AUCs, and these indices were the most important parameters [26]. ROC AUCs of bandwidth, phase SD, and entropy showed good diagnostic accuracy with values $\geq 0.850$ in our results.

\section{Acquisition condition and gamma camera}

The Japanese normal database consists of 122 normal patients enrolled from four hospitals. Various gamma cameras with LEHR and Vertex general-purpose (VXGP) collimators manufactured by ADAC, Elscint, Toshiba, and SIEMENS were used in the four hospitals. 

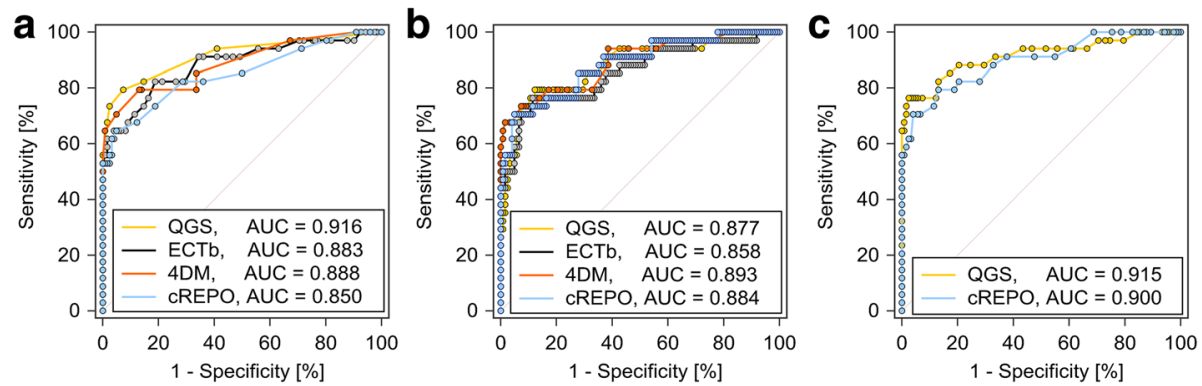

Fig. 6 ROC curves of bandwidth (a), phase SD (b), and entropy (c) in QGS, ECTb, 4DM, and CREPO. ROC receiver operator characteristics, SD standard deviation, AUC area under the ROC curve, QGS quantitative gated SPECT, ECTb Emory Cardiac Toolbox, 4DM Corridor 4DM, CREPO cardioREPO

The acquisition conditions of myocardial perfusion SPECT were essentially the same in the four hospitals. All gated SPECT acquisitions were performed using $64 \times 64$ matrix with 16 frames per RR interval. However, 180 or $360^{\circ}$ circular rotation of gamma cameras with step and shoot mode was used. When we calculated the normal bandwidth and phase SD using the ECTb software program derived from 180 or $360^{\circ}$ SPECT acquisition in the four hospitals, there were no significant differences in bandwidth $\left(30.0^{\circ} \pm 8.5^{\circ}\right.$ vs. $27.2^{\circ} \pm 9.3^{\circ} ; p=$ $0.11)$ and phase SD $\left(11.6^{\circ} \pm 5.1^{\circ}\right.$ vs. $\left.9.8^{\circ} \pm 4.5^{\circ} ; p=0.07\right)$. Furthermore, when we additionally computed bandwidth and phase SD using the ECTb software program in each hospital, there were no significant differences among the four hospitals in bandwidths $(30.9 \pm 9.0,30.0 \pm 9.3,29.0$ \pm 6.9 , and $27.2 \pm 9.3 ; p=0.43$ by analysis of variance (ANOVA) $)$ and phase $(10.4 \pm 3.5,11.8 \pm 6.3,12.4 \pm 4.8$, and $9.8 \pm 4.5 ; p=0.20$ by ANOVA). Although there were no significant differences among four hospitals in normal bandwidth and phase SD, further clinical validation might be required to determine whether to harmonize the acquisition methodologies and gamma cameras in multicenter study or not.

\section{Limitation}

We only performed phase analysis of rest GMPS in this study. Since myocardial perfusion count statistics vary under stress and rest conditions, ROC AUCs and cutoff values will be different under stress conditions. Furthermore, sex differences in bandwidth, phase SD, and entropy have been reported $[7,23,30,31]$. The sexspecific cutoff values of phase parameters might clearly discriminate between normal patients and patients with suspected LV dyssynchrony. Moreover, further investigation would be required to determine the diagnostic accuracy of CRT using optimal cutoff values and to evaluate the effect of CRT on improvement of dyssynchrony in new follow-up study after the treatment. Although this study used a multicenter database in normal patients, patients with suspected LV dyssynchrony were enrolled in a single center; thus, multicenter validation should be conducted.

\section{Conclusions}

The mean bandwidth, phase SD, and entropy significantly differed in 4DM, cREPO, ECTb, and QGS software programs in patients with normal perfusion and
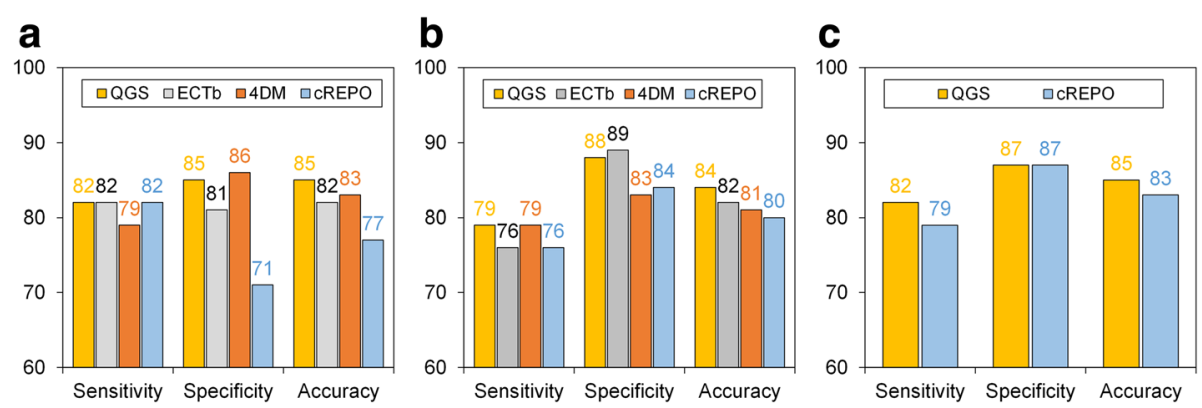

Fig. 7 The sensitivity, specificity, and accuracy of ROC analysis in bandwidth (a), phase SD (b), and entropy (c). The sensitivity, specificity, and accuracy were computed by QGS, ECTb, 4DM, and CREPO. ROC receiver operator characteristics, SD standard deviation, QGS quantitative gated SPECT, ECTb Emory Cardiac Toolbox, 4DM Corridor 4DM, CREPO cardioREPO 
Table 2 Cutoff points for phase parameters in the four software programs

\begin{tabular}{lllll}
\hline & QGS & ECTb & 4DM & CREPO \\
\hline Bandwidth $\left(^{\circ}\right)$ & 24 & 35 & 36 & 42 \\
Phase SD $\left(^{\circ}\right)$ & 8.6 & 15.3 & 9.4 & 11.9 \\
Entropy $(\%)$ & 31 & - & - & 48
\end{tabular}

Abbreviations: 4DM Corridor 4DM, CREPO cardioREPO, ECTb Emory Cardiac Toolbox, QGS quantitative gated SPECT

cardiac function. The LV dyssynchrony parameters of bandwidth, phase SD, and entropy obtained by 4DM, cREPO, and QGS were highly correlated with those by ECTb. Although the optimal cutoff values of bandwidth, phase SD, and entropy were variable depending on the software programs, the diagnostic performance by ROC analysis was equivalent. All four software programs can be used reliably for phase analysis in GMPS.

\section{Abbreviations}

4DM: Corridor4DM; ${ }^{99 m}$ Tc MIBI: Technetium-99m methoxy-isobutyl-isonitrile: BBB: Bundle branch block; cREPO: CardioREPO; CRT: Cardiac resynchronization therapy; ECTb: Emory Cardiac Toolbox; HF: Heart failure; QGS: Quantitative gated SPECT; SPECT: Single-photon emission computed tomography

\section{Acknowledgements}

This study was performed as part of the JSNM-WG activity of "Creation of common databases in nuclear cardiology and cross-calibration among software programs (2007 and 2015)". We would like to thank the following for using commercially available software packages: ECTb with SyncTool by Syntermed, Inc., (Atlanta, GA, USA) and Corridor4DM by INVIA Medical Imaging Solutions (Ann Arbor, MI, USA). We also thank nuclear medicine technologists of Kanazawa University Hospital, Japan, for their assistance, and Karin Toth of EXINI Diagnosis, Lund, Sweden, for preparing the phase data for CREPO, and Editage (www.editage.jp), for English language editing.

\section{Funding}

This study was partly funded by the JSPS KAKENHI grants (numbers 26861022 and 15 K09947) as well as the grants for Promoted Research from the Kanazawa Medical University (S2014-13 and S2016-6)

\section{Authors' contributions}

$\mathrm{KO}$ drafted the manuscript, and $\mathrm{KN}$ edited it. $\mathrm{KO}, \mathrm{SM}$, and SK performed data analysis and interpretation. KO performed the statistical analysis of the data, and KN confirmed it. HY, TS, and MO provided technical support for phase analysis. KN, MH, and SK participated in the conception and study design. All authors read and approved the final manuscript.

\section{Competing interests}

K Nakajima has performed a collaborative research work with FUJIFILM RI Pharma Co. Ltd, Tokyo, Japan, which developed the cardioREPO software program. The other authors declare that they have no competing interests.

\section{Publisher's Note}

Springer Nature remains neutral with regard to jurisdictional claims in published maps and institutional affiliations.

\section{Author details}

'Department of Physics, Kanazawa Medical University, 1-1 Daigaku, Uchinada, Kahoku, Ishikawa 920-0293, Japan. ²Department of Nuclear Medicine, Kanazawa University Hospital, 13-1 Takara-machi, Kanazawa, Ishikawa 920-8641, Japan. ${ }^{3}$ Department of Radiological Technology, Kanazawa Municipal Hospital, Kanazawa, Japan. ${ }^{4}$ Department of Radiological Technology, Kanazawa University Hospital, Kanazawa, Japan. ${ }^{5}$ Department of Quantum Medical Technology, Kanazawa University, Kanazawa, Japan.
Received: 22 December 2016 Accepted: 8 March 2017

Published online: 23 March 2017

\section{References}

1. Hunt SA, Abraham WT, Chin MH, Feldman AM, Francis GS, Ganiats TG, et al. 2009 Focused update incorporated into the ACC/AHA 2005 Guidelines for the Diagnosis and Management of Heart Failure in Adults: a report of the American College of Cardiology Foundation/American Heart Association Task Force on Practice Guidelines developed in collaboration with the International Society for Heart and Lung Transplantation. J Am Coll Cardiol. 2009;53:e1-e90.

2. Swedberg K, Cleland J, Dargie H, Drexler H, Follath F, Komajda M, et al. Guidelines for the diagnosis and treatment of chronic heart failure: executive summary (update 2005). Eur Heart J. 2005;26:1115-40. The Task Force for the diagnosis and treatment of chronic heart failure of the European Society of Cardiology.

3. Bax JJ, Bleeker GB, Marwick TH, Molhoek SG, Boersma E, Steendijk P, et al. Left ventricular dyssynchrony predicts response and prognosis after cardiac resynchronization therapy. J Am Coll Cardiol. 2004;44:1834-40.

4. Yu CM, Fung WH, Lin H, Zhang Q, Sanderson JE, Lau CP. Predictors of left ventricular reverse remodeling after cardiac resynchronization therapy for heart failure secondary to idiopathic dilated or ischemic cardiomyopathy. Am J Cardiol. 2003;91:684-8

5. Westenberg JJ, Lamb HJ, van der Geest RJ, Bleeker GB, Holman ER, Schalij $\mathrm{MJ}$, et al. Assessment of left ventricular dyssynchrony in patients with conduction delay and idiopathic dilated cardiomyopathy: head-to-head comparison between tissue doppler imaging and velocity-encoded magnetic resonance imaging. J Am Coll Cardiol. 2006:47:2042-8.

6. White JA, Yee R, Yuan X, Krahn A, Skanes A, Parker M, et al. Delayed enhancement magnetic resonance imaging predicts response to cardiac resynchronization therapy in patients with intraventricular dyssynchrony. $J$ Am Coll Cardiol. 2006;48:1953-60

7. Chen J, Garcia EV, Folks RD, Cooke CD, Faber TL, Tauxe EL, et al. Onset of left ventricular mechanical contraction as determined by phase analysis of ECG-gated myocardial perfusion SPECT imaging: development of a diagnostic tool for assessment of cardiac mechanical dyssynchrony. J Nucl Cardiol. 2005:12:687-95.

8. Zafrir N. Left ventricular mechanical dyssynchrony graduation of myocardial perfusion gated SPECT phase analysis: What next. J Nucl Cardiol. 2016:1-3. Epub ahead of print

9. Zhou W, Jiang Z, Chen J, Garcia EV, Li D. Development and validation of a phase analysis tool to measure interventricular mechanical dyssynchrony from gated SPECT MPI. J Nucl Cardiol. 2016. (Epub ahead of print)

10. Kiso K, Imoto A, Nishimura Y, Kanzaki H, Noda T, Kamakura S, et al. Novel algorithm for quantitative assessment of left ventricular dyssynchrony with ECG-gated myocardial perfusion SPECT: useful technique for management of cardiac resynchronization therapy. Ann Nucl Med. 2011;25:768-76.

11. Garcia EV, Faber TL, Cooke CD, Folks RD, Chen J, Santana C. The increasing role of quantification in clinical nuclear cardiology: the Emory approach. J Nucl Cardiol. 2007:14:420-32.

12. Germano G, Kavanagh PB, Slomka PJ, Van Kriekinge SD, Pollard G, Berman DS. Quantitation in gated perfusion SPECT imaging: the Cedars-Sinai approach. J Nucl Cardiol. 2007:14:433-54

13. Ficaro EP, Lee BC, Kritzman JN, Corbett JR. Corridor4DM: the Michigan method for quantitative nuclear cardiology. J Nucl Cardiol. 2007;14:455-65.

14. Nakajima K, Matsuo S, Wakabayashi H, Yokoyama K, Bunko H, Okuda K, et al. Diagnostic performance of artificial neural network for detecting ischemia in myocardial perfusion imaging. Circ J. 2015;79:1549-56.

15. Johansson L, Edenbrandt L, Nakajima K, Lomsky M, Svensson SE, Tragardh E. Computer-aided diagnosis system outperforms scoring analysis in myocardial perfusion imaging. J Nucl Cardiol. 2014;21:416-23.

16. Edenbrandt L, Hoglund P, Frantz S, Hasbak P, Johansen A, Johansson L, et al. Area of ischemia assessed by physicians and software packages from myocardial perfusion scintigrams. BMC Med Imaging. 2014;14:5.

17. Nakajima K, Okuda K, Nystrom K, Richter J, Minarik D, Wakabayashi H, et al. Improved quantification of small hearts for gated myocardial perfusion imaging. Eur J Nucl Med Mol Imaging. 2013;40:1163-70.

18. Yoneyama $\mathrm{H}$, Nakajima K Okuda K, Matsuo S, Onoguchi M, Kinuya S et al. Reducing the small-heart effect in pediatric gated myocardial perfusion single-photon emission computed tomography. J Nucl Cardiol. 2016. (Epub ahead of print) 
19. Nakajima K, Matsumoto N, Kasai T, Matsuo S, Kiso K, Okuda K. Normal values and standardization of parameters in nuclear cardiology: Japanese Society of Nuclear Medicine working group database. Ann Nucl Med. 2016:30:188-99.

20. Nakajima K. Normal values for nuclear cardiology: Japanese databases for myocardial perfusion, fatty acid and sympathetic imaging and left ventricular function. Ann Nucl Med. 2010;24:125-35.

21. Nakajima K, Kumita S, Ishida Y, Momose M, Hashimoto J, Morita K, et al. Creation and characterization of Japanese standards for myocardial perfusion SPECT: database from the Japanese Society of Nuclear Medicine Working Group. Ann Nucl Med. 2007;21:505-11.

22. Akhter N, Nakajima K, Okuda K, Matsuo S, Yoneyama T, Taki J, et al. Regional wall thickening in gated myocardial perfusion SPECT in a Japanese population: effect of sex, radiotracer, rotation angles and frame rates. Eur J Nucl Med Mol Imaging. 2008;35:1608-15.

23. Van Kriekinge SD, Nishina H, Ohba M, Berman DS, Germano G. Automatic global and regional phase analysis from gated myocardial perfusion SPECT imaging: application to the characterization of ventricular contraction in patients with left bundle branch block. J Nucl Med. 2008;49:1790-7.

24. Youden WJ. Index for rating diagnostic tests. Cancer. 1950;3:32-5.

25. Atchley AE, Trimble MA, Samad Z, Shaw LK, Pagnanelli R, Chen J, et al. Use of phase analysis of gated SPECT perfusion imaging to quantify dyssynchrony in patients with mild-to-moderate left ventricular dysfunction. J Nucl Cardiol. 2009;16:888-94.

26. Romero-Farina G, Aguade-Bruix S, Candell-Riera J, Pizzi MN, Garcia-Dorado D. Cut-off values of myocardial perfusion gated-SPECT phase analysis parameters of normal subjects, and conduction and mechanical cardiac diseases. J Nucl Cardiol. 2015;22:1247-58.

27. Trimble MA, Borges-Neto S, Smallheiser S, Chen J, Honeycutt EF, Shaw LK, et al. Evaluation of left ventricular mechanical dyssynchrony as determined by phase analysis of ECG-gated SPECT myocardial perfusion imaging in patients with left ventricular dysfunction and conduction disturbances. J Nucl Cardiol. 2007;14:298-307

28. Trimble MA, Velazquez EJ, Adams GL, Honeycutt EF, Pagnanelli RA, Barnhart $H X$, et al. Repeatability and reproducibility of phase analysis of gated singlephoton emission computed tomography myocardial perfusion imaging used to quantify cardiac dyssynchrony. Nucl Med Commun. 2008;29:374-81.

29. AlJaroudi W, Jaber WA, Cerqueira MD. Effect of tracer dose on left ventricular mechanical dyssynchrony indices by phase analysis of gated single photon emission computed tomography myocardial perfusion imaging. J Nucl Cardiol. 2012;19:63-72.

30. Hamalainen H, Hedman M, Laitinen T, Hedman A, Kivela A, Laitinen T. Reference values for left ventricular systolic synchrony according to phase analysis of ECG-gated myocardial perfusion SPECT. Clin Physiol Funct Imaging. 2016. (Epub ahead of print)

31. Nakajima K, Okuda K, Matsuo S, Kiso K, Kinuya S, Garcia EV. Comparison of phase dyssynchrony analysis using gated myocardial perfusion imaging with four software programs: based on the Japanese Society of Nuclear Medicine working group normal database. J Nucl Cardiol. 2016. (Epub ahead of print)

\section{Submit your manuscript to a SpringerOpen ${ }^{\circ}$ journal and benefit from:}

- Convenient online submission

- Rigorous peer review

- Immediate publication on acceptance

- Open access: articles freely available online

- High visibility within the field

- Retaining the copyright to your article 